\title{
Transcending the Self in Immersive Virtual Reality
}

\author{
Mel Slater ${ }^{1,2,3^{*}}$ \& Maria V. Sanchez-Vives ${ }^{1,2,4}$ \\ ${ }^{1}$ ICREA, Passeig Lluís Companys 23, Barcelona 08010, Spain \\ ${ }^{2}$ Facultat de Psicologia, Universitat de Barcelona, Campus de \\ Mundet - Edifici Teatre, Passeig de la Vall d'Hebron \\ 171, Barcelona 08035, Spain \\ ${ }^{3}$ Department of Computer Science, University College London \\ ${ }^{4}$ Institut d'Investigacions Biomèdiques August Pi i Sunyer, Rosselló, \\ 149-153, Barcelona 08036, Spain
}

* Corresponding author: melslater@ub.edu

\begin{abstract}
Cognitive neuroscientists have discovered various experimental setups that suggest that our body representation is surprisingly flexible, where the brain can easily be tricked into the illusion that a rubber hand is your hand or that a manikin body is your body. These multisensory illusions work well in immersive virtual reality (IVR). What is even more surprising is that such embodiment induces perceptual, attitudinal and behavioural changes that are concomitant with the displayed body type. Here we outline some recent findings in this field, and suggest that this offers a powerful tool for neuroscience, psychology and a new path for IVR.
\end{abstract}

\section{Introduction}

In immersive virtual reality it is possible to give people the illusion that they have a different body. They wear a head-tracked head-mounted display, and when they look down towards themselves they see a virtual body that is spatially coincident with their real body. Through realtime motion capture when the person moves their real body they see the virtual body move correspondingly. They can also see this in a virtual mirror reflection (and shadows) of their body as well as looking directly towards it [1].

Since the virtual reality is entirely programmed, the form or type of virtual body can be quite different to the person's real body and the type of body can have an impact on perception, attitudes and behaviors of the participant. Our most dramatic example of this is that when adults are embodied in a virtual body depicting a child they overestimate the size of objects, and have implicit attitude and behavioral changes towards becoming child-like. When they are placed in an adult body that is the same size as the child one, then they do not exhibit such changes [2]. In this

${ }^{*}$ Note that this is not the final published version which is available on

http://ieeexplore.ieee.org/xpl/articleDetails.jsp?arnumber $=6861900$ 
paper we review the emerging field of body representation and its implications for a new powerful virtual reality paradigm. First we briefly address how virtual reality has been typically use to transform people's sense of place, and then consider how it can be applied to transform the self.

\section{Immersive Virtual Reality and Presence}
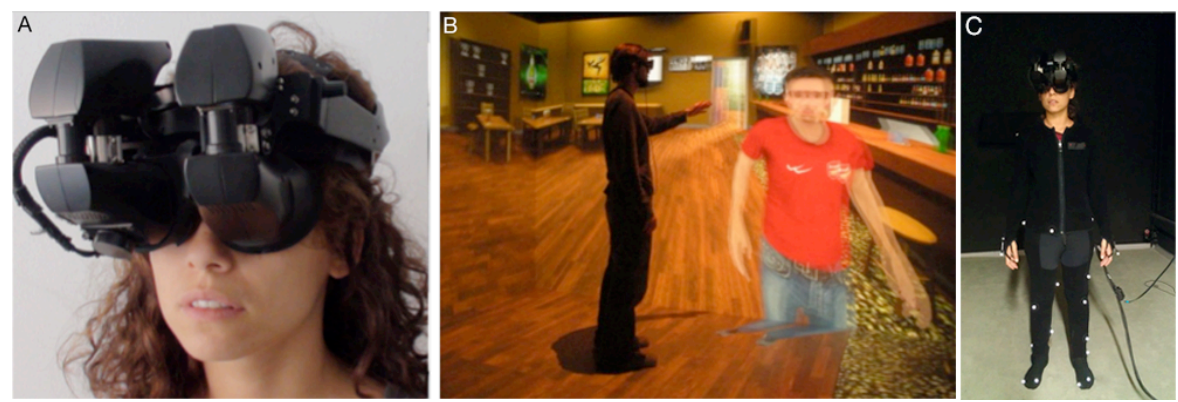

Figure 1 - Major types of IVR System. (A) A head mounted display. (B) A person is interacting with a virtual human in a Cave system. The double image can be seen in the photo since this is a stereo display. The participant wears head-tracked glasses to merge the double image into a 3D surrounding environment.

A standard IVR system has two critical components. The first is a display device that delivers surrounding high-resolution stereovision and sound to a participant, that substitutes their real world sensory input by computer generated input, including haptic feedback. The second is the use of tracking so that at the very least their head is tracked (with six degrees of freedom) in real time. The result of this is that the world is displayed to the participant depending on head gaze direction. There are two major forms of delivery. The first is a head-mounted display (HMD) that deliver wide field-of-view stereoscopic vision, and on which is mounted the head-tracking device (Figure 1A). The second is a projection based system - typically called a Cave - where the walls of a room are back projection stereo display screens, and the participant wears shutter glasses so that each eye only receives images that correspond to the image for that eye (Figure 1B). Again the glasses typically incorporate a head-tracker. In the type of research discussed in this paper there is a third critical system, which is a full body motion capture suit. This tracks body movements of the participant that is used to drive in real time the virtual body that substitutes the real body.

There is substantial evidence gathered over many years that when people enter an immersive virtual reality (IVR) they tend to act as if they were in a real place and as if the events there were really happening. This type of response has generally been referred to as 'presence' (see [3] for a review). Originally this was simply thought of as the sensation of 'being there' in the virtual environment, but more recent interpretations focus on the extent to which people respond realistically, with a fundamental distinction between the illusion of being in a place ("place illusion'), and experiencing events as if they were real ('plausibility') [4]. Place illusion relies heavily on head (and ideally eye tracking) since this affords head-based sensorimotor contingencies - i.e., perception of the environment through the use of the body in a natural way (bending down, looking around, looking past, listening by turning the head towards the source). The Plausibility Illusion (Psi) is the extent to which the events within the virtual environment are perceived as really happening. This seems to be a function of three affordances of the environment (a) the extent to which events in the environment refer specifically to the participant (b) the extent to which there are events that respond to the actions of the person (e.g., the participant smiles at a virtual human that smiles back) and (c) the overall credibility of the 
environment in comparison to expectations. An example where PI and Psi came together to result in behavioral and physiological responses of participants that were close to matching experiments that had been carried out in reality was the virtual reprise of the famous 1960s Stanley Milgram obedience experiments [5].

Thus IVR has been used extensively to transform the 'place' and 'events' in which people are illusorily involved. Here we consider a different (but related) application of IVR: transforming the self.

\section{Body Ownership Illusions}

In the late 1980s Jaron Lanier experimented with the idea of using virtual reality to transform the self through endowing participants with virtual bodies. Writing about these experiences Lanier observed that "It turned out that people could quickly learn to inhabit strange and different bodies and still interact with the virtual world. I became curious about how weird the body could get before the mind would become disoriented. I played around with elongated limb segments and strange limb placements. The most curious experiment involved a virtual lobster...." [6](p122). What he called 'homuncular flexibility' meant that people can learn to inhabit and naturally control virtual bodies that are quite different to their own.

These observations about the flexibility of the brain attributing surrogate bodies or body parts to the self (such as virtual bodies) were unpublished and remained unnoticed and unexplored in the scientific literature. However, research over the last two decades in cognitive neuroscience has indeed confirmed that the brain is remarkably plastic with respect to body representation. The rubber hand illusion (RHI) [7] shows that by tapping and stroking a rubber hand placed in an anatomically plausible position on a table in front of a person, and synchronously tapping and stroking the corresponding but hidden real hand, a strong illusion that the rubber hand is their hand quickly develops. This illusion is both subjective and can be measured objectively through 'proprioceptive drift' meaning that when asked to blindly point towards their hand they will point more towards the rubber than the real hand. Similarly, if the rubber hand is seen to be threatened, then there are strong physiological responses to the perceived threat [8]. The illusion of ownership and objective indicators occur to a significantly lesser effect when the visual and tactile stimuli are not both temporally and spatially synchronous.

This illusion has been shown to work well in immersive virtual reality, where instead of a rubber arm, a virtual arm is seen in stereo 3D as coming out of the real shoulder of the experimental participant [9]. The synchronous visual-tactile stimulation was achieved by the experimental operator using a tracked Wand to tap and stroke the participant's real hidden hand, while a virtual ball controlled by the Wand was seen to effect the same touch on the virtual hand. Petkova and Ehrsson demonstrated that the same principles of multisensory stimulation can result in the illusion of ownership over a whole manikin body seen from first person perspective [10], which we showed also functions very well in IVR [1].

\section{The Consequences of Body Ownership Illusions}

Returning to the introduction - a person's whole body can be 'replaced' by a virtual body in IVR so when they look down towards their own body, they would see the virtual body instead. Additionally when they look towards a virtual mirror they would see this virtual body reflected back. This is already a very powerful cue to the brain to feel this virtual body as their own since throughout life whenever we look down towards our body - or in a mirror - of course we see our own body. Hence first person perspective (1PP) (the eyes of the virtual body coincident with the person's real eyes, and the virtual and real body being spatially coincident) is a very powerful factor towards inducing a whole body ownership illusion [1], [11]. In such setups there is real- 
time motion capture of the participant's movements. Hence any movement of their real body can be mapped onto movements of the virtual body.
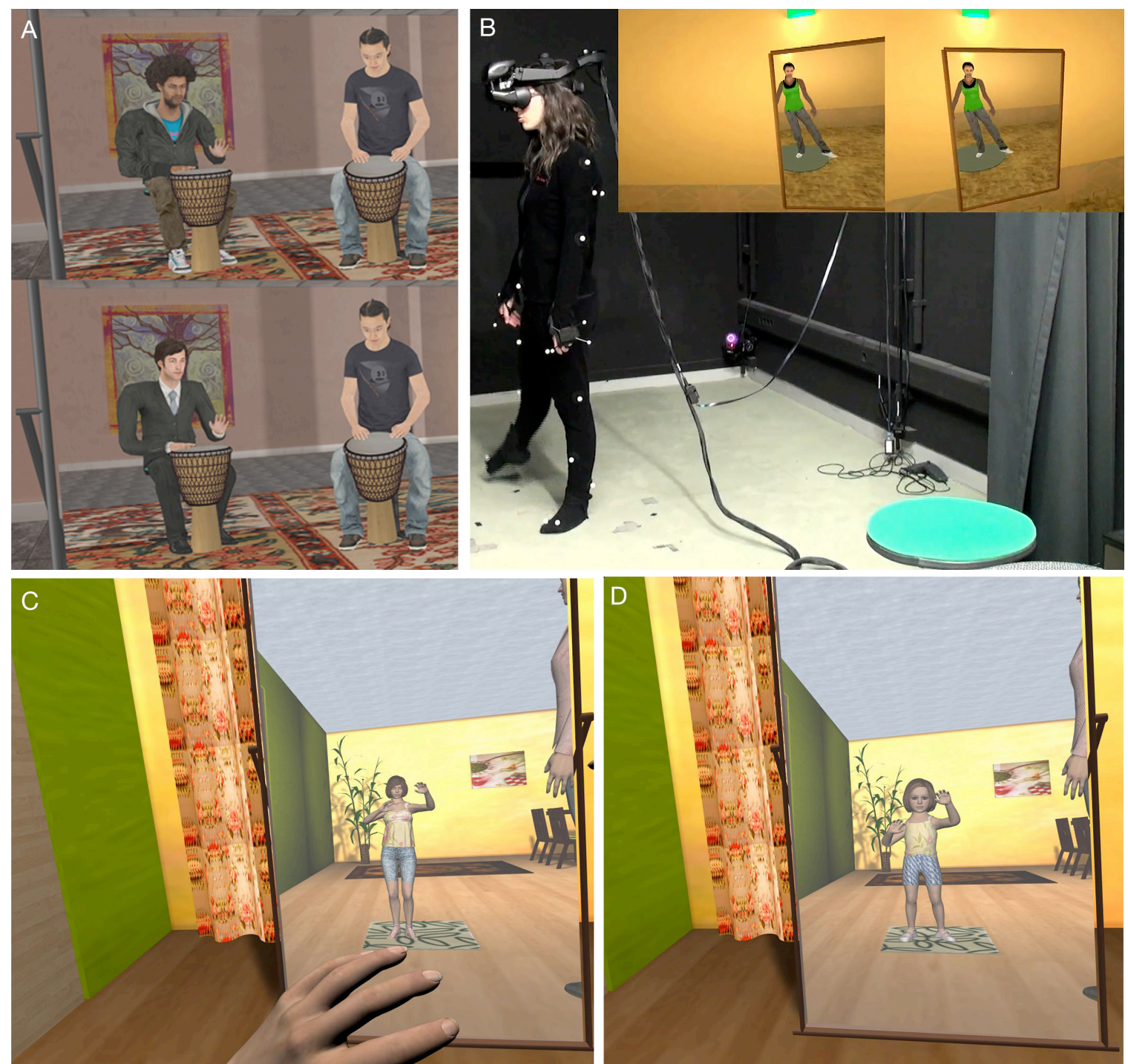

Figure 2 - Embodiment examples (A) The drumming experiment view in the virtual mirror of the Casual Dark Skinned condition (top) and Formal Light-Skinned (bottom). (B) The dark-skinned avatar in the racial bias experiment where avatar's movements are driven by those of the participant (C) The adult embodiment experiment where the avatar shown is the scaled down adult one (D) the child embodiment where the avatar is the child one.

We use the term 'embodiment' to refer to this setup - where a virtual body is spatially coincident with your real body, you see through the eyes of that virtual body, and with various types of synchronous multisensory correlation. We describe how to achieve this technically in [12].

Here we ask - suppose you have the perceptual illusion that an alternate virtual body is yours, what implications might this have for behavior, attitudes, and cognition, both within the virtual reality and beyond? This issue has been investigated under a paradigm known as the 'Proteus Effect' [13], where it was argued that the digital self representation of a person could influence their attitudes and behaviors in online and virtual environments. Essentially the personality 
associated with the digital representation would influence the actual real-time behaviors of the participants.

The idea of 'Proteus' is that of mutability. We prefer a term that suggests not just mutability but that the form carries information and meaning. Hence we have used the term 'body semantics' to express the finding that when through appropriate multisensory correlations participants have the illusion of ownership and agency over a virtual body (or indeed a robotic one) then this process has behavioral, attitudinal and probably also cognitive correlates for the embodied person. Here we consider three recent examples that illustrate this.

\section{Motor Actions}

How much might the motor actions of a person be influenced by the type of virtual body in which they are embodied? We considered this question in the context of drumming performance [14].

Thirty six people took part in an experiment (17 males) where they were required to play a Djembe hand drum in an IVR. The participants were divided into two different groups. The Casual Dark-Skinned (CD) group saw a virtual body that was dressed casually and was darker skinned (than all the participants). Those in the other group were represented in a Formal LightSkinned body (FL). All were accompanied by an animated virtual drummer (Figure 2A) ${ }^{1}$. Throughout their experience we recorded 36 data points from the motion capture, representing the movement of the upper body. This was recorded first in a baseline condition where the participants were represented solely by non-textured white hands (i.e., without a body) and then during the actual embodiment period. To estimate the amount of movement we used a principle components analysis to compute the number of dimensions needed to capture $95 \%$ of the total variance in the complete data.

There were no significant differences in the subjective level of body ownership between the two groups, as measured by a questionnaire, and the questionnaire scores indicated a strong ownership illusion. The results showed, however, that those in the CD group required on average more than one dimension more during the embodiment period compared to the baseline period to represent $95 \%$ of the variance of the motion capture data $(\mathrm{P}=0.0002)$, whereas for those in the FL condition there was no difference from baseline $(\mathrm{P}=0.90)$. Overall those in the CD group exhibited significantly greater upper body movement than those in the FL group, and the amount of movement was positively correlated with the extent of subjective body ownership. Hence solely the type of body (including clothes, hairstyle etc) influenced the motor performance.

\section{Racial Bias}

Using the same virtual reality setup as described above, we studied racial bias. The objective was to examine whether deep seated implicit attitudes could be shifted by virtual embodiment [15]. In this experiment 60 females experienced one of 4 conditions (15 participants per condition in a between-groups design). In the Embodied Dark (ED) condition they were self-represented as a dark-skinned female avatar (Figure 2B); in the Embodied Light (EL) as a light-skinned avatar; in an Embodied Alien (EA) condition they were embodied in a purple skinned body, and finally in a Non-embodied Dark (ND) condition they had no direct body representation, but could see a reflection of a dark-skinned body in a virtual mirror that had movements independent of their own body movements.

Some days before the virtual reality experience participants attended the laboratory and completed a racial bias Implicit Association Test (IAT) [16]. This type of test measures bias by response time when categorizing relationships between concepts. For example, a bias against black people is indicated when reaction times are faster in associating white faces with positive words and black faces with negative words than white faces with negative words and black faces

${ }^{1}$ A video is available on https://www.youtube.com/watch?v=ydzSgLim5Y4 
with positive words. In the IVR experience participants were embodied altogether for approximately 12 minutes in one of the 4 situations described above. For 7 of those minutes 12 virtual characters walked by the participant, half of these black and half of them white, and simply looked at the participant while walking past ${ }^{2}$. At the end of this participants carried out the IAT test again, and also answered a questionnaire.

While an explicit racial bias test showed on the average a low degree of racial bias, the initial IAT test did show implicit racial bias. However, the effect after the experiment was different between the four groups. The reduction in the mean level of racial bias as a result of the exposure was greater in the ED group compared with the EL group $(\mathrm{P}=0.03)$. The difference between the ED group and the others was striking when a covariate signifying presence was taken into account. (The factor that represented presence was the degree of their reported nervousness as the avatars walked past - breaking into their personal space). Analysis of Covariance of the change in IAT on condition (ED, EL, EA, ND) with nervousness as the covariate showed a negative slope indicating reduced racial bias with increasing nervousness $(\mathrm{P}=0.002)$ and with ED having a significantly lower intercept than EL $(\mathrm{P}=0.009)$ but no other significant differences.

The level of subjective body ownership as measured by questionnaire responses was not different between the ED, EL and EA groups but significantly lower in the ND condition. The overall ANOVA showing a difference between the mean levels of ownership had significance level $\mathrm{P}=0.004$. All pairwise comparisons at an overall significance level of $5 \%$ showed that the mean level of ND was less than each of the others, but that there were no differences between the embodiment conditions. Moreover in the embodiment conditions the mean levels of body ownership were high (approximately 4 on a 1 to 5 Likert scale, with 1 meaning the least and 5 the greatest levels). Hence it was the difference in body types (specifically skin color) that was responsible for the reduction in implicit racial bias in the ED group.

\section{Being a Child}

Here we considered whether embodiment in a young child body would influence perception of object sizes and attitudes about the self [2]. In a first experiment 30 adults were embodied in a child body (approximately 4 years old) or in an adult body of the same size as the child (Figure 2C-D). Hence the only difference between the two conditions was the shape of the virtual body (either child or adult). There was whole body motion capture so that the virtual body movements were the same as their $\mathrm{own}^{3}$. Participants were asked before and after the experience to indicate the sizes of a number of objects by the distance between their hands. There were no differences between the adult and child conditions with respect to the subjective illusion of body ownership, which was high. As expected, based on previous results, both groups overestimated object sizes. However compared to the baseline readings, the child condition estimates were almost double those of the adult condition estimates. For example, the differences in mean estimates for the three object sizes tested all had significance levels $\mathrm{P}<0.006$. Moreover the IAT results showed the child condition resulting in significantly greater implicit association of the self with child-like categories than the adult condition $(\mathrm{P}=0.0001)$.

In order to be sure that the results really reflected body ownership, we ran a second experiment with a further 16 participants. This was the same as the first, except that the virtual body moved independently of the real body movements of the person. However, the body was still seen from 1PP and in the mirror as before, and all else was the same as in the first experiment. The subjective level of body ownership then was very low. Both groups overestimated object sizes to about the same extent as those in the adult condition in the first experiment, but there were no differences between the adult and child condition and there was no difference in the IAT scores. Hence the introduction of body movements that did not match the

${ }^{2}$ A video is available on https://www.youtube.com/watch?v=HliN3iOX090

${ }^{3} \mathrm{~A}$ video is available on https://www.youtube.com/watch?v=80y830VgbSM 
real body movements extinguished body ownership and agency, and all of the differences between the child and adult conditions.

\section{Discussion}

The field of body representation raises several very interesting issues for virtual reality. The first is that it is excellent as a practical tool for cognitive neuroscience to study how the brain represents the body. It offers a flexibility for the manipulation of body representation that far surpasses the typical tools of the trade such as rubber arms, mirrors and LEDs. Everything is fully programmable so that highly sophisticated manipulations can be achieved. Moreover, the cost of setting up a VR laboratory is declining dramatically.

Second, IVR is helping to uncover the striking plasticity of the brain with respect to body representation. Jaron Lanier had this insight decades ago, but this was never followed-up scientifically. Cognitive neuroscience through a completely different paradigm has come to the same conclusions.

Third, and perhaps most interesting of all is the idea of 'body semantics' - that the brain is apparently able to drive attitudes and behavior of an individual according to their level of body ownership with respect to the type virtual body in which they are embodied. Although the three studies that we have presented are apparently quite different, they are closely linked through the idea that the form or type of the body over which a participant has an illusion of ownership, can influence - their motor behavior (the first experiment), their implicit racial bias (the second experiment), their perception of object sizes and self-categorization as child- or adult-like (the third experiment). These are all examples of what we have termed 'body semantics' - the body form influencing various aspects of perception, attitudes and behavior.

It is worth while emphasizing both the similarities and differences between the 'Proteus Effect' [13] and 'body semantics'. The similarity is that both claim (and have demonstrated) that the type of body can influence attitudes and behaviors. However, this conclusion is arrived at through quite different paradigms. The Proteus Effect is from a social psychological perspective based on the idea that people behave according to how others would expect someone with that type of body to behave. It is based on 'self perception theory', where participants 'conform to the behavior that they believe others would expect them to have' (p274), and that '... self-perception theory is not predicated on the actual presence of other people but simply that a person evaluates him or herself from a third-person perspective (i.e., an imagined third party)' (p274-5).

The idea of 'body semantics' is that when the brain generates an illusion of body ownership and agency over a virtual body then a correlate of this is that it also generates attitudes and behaviors that are concomitant with that type of body, independently of any other factors such as social expectation. This is an intrinsic property of brain functioning, and not necessarily a product of social expectation, whether actual or imagined. The interesting and quite unresolved question though is how the brain derives those attitudes and behaviors. In the case of the child it could simply be that we have all been children, and hence there is a reliance on autobiographical memory to determine perception and other mental processing. However, none of the participants in our drumming experiment had once been drummers who looked something like Jimmy Hendrix. So it is possible that the brain here is relying on statistical information about how people with such bodies that they have seen in the past behaved in that setting. Or perhaps independently of statistical information gathered from living in a particular society and culture, brains have intrinsic mechanisms that encapsulate extremely tight associations between the type of body and the behaviors of which it is capable. 


\section{Conclusions}

Body representation and body semantics opens up new possibilities for application of IVR. An obvious one is concerned with pain. If a person experiencing pain or discomfort is embodied in a virtual body that indicates health, how much might this alleviate pain? Some results have been shown in this regard [17], [18], and of course it could apply beyond pain to other medical and psychological conditions. Generally, body semantics opens up the possibility to experience the world from a different point of view - to have feelings, for example, of how it might be to be a child, or how it might feel to be a member of a minority group.

Although we have limited our discussion to embodiment in virtual bodies, most of the same considerations apply to embodiment in robots. The relatively young field of neuroprosthetics is concerned with giving people artificial limbs that they control through brain implants or braincomputer interfaces. Using the principles of multisensory feedback for body ownership and agency, it should be possible also to give people a sense of somatic ownership over such artificial body parts or over a whole virtual body [19].

Our final point is that virtual reality has mainly been thought of as a way to relocate people to virtual places and take part in events and activity there. Here we demonstrate the likelihood of far reaching future applications where the concentration is not on changing the place, but changing the self. Through alternate virtual bodies people can have experiences and gain access to resources locked up in their brain that may help them in many aspects of their lives.

\section{Acknowledgments}

The work described in this paper has been funded under the following projects: European Research Council ERC Advanced Grant TRAVERSE (\#227985), the European Union FP7 Future and Emerging Technologies (FET) Integrated Project VERE (\#257695), the European Union FP7 ICT Integrated Project BEAMING (\#248620), the FP7 European Union AAT Program project VR-HYPERSPACE (\#AAT-285681) and the Spanish Ministerio de Ciencia i Innovación project ResPIVE (\#PSI2011-27930). We would also like to thank all people working in the Event Lab, University of Barcelona that makes this work possible. 


\section{References}

[1] M. Slater, B. Spanlang, M. Sanchez-Vives, and O. Blanke, "First person experience of body transfer in virtual reality," PLoS One, vol. 5, no. 5, p. e10564, 2010.

[2] D. Banakou, R. Groten, and M. Slater, "Illusory ownership of a virtual child body causes overestimation of object sizes and implicit attitude changes," PNAS, vol. 110, no. 31, pp. 12846$12851,2013$.

[3] M. V Sanchez-Vives and M. Slater, "From Presence to Consciousness Through Virtual Reality," Nat.Rev. Neurosci., vol. 6, no. 4, pp. 332-339, 2005.

[4] M. Slater, "Place Illusion and Plausibility can lead to realistic behaviour in immersive virtual environments," Philos Trans R Soc L., vol. 364, no. 1535 , pp. 3549-3557, 2009.

[5] M. Slater, A. Antley, A. Davison, D. Swapp, C. Guger, C. Barker, N. Pistrang, and M. V SanchezVives, "A virtual reprise of the Stanley milgram obedience experiments," PLoS One, vol. 1, no. 1, p. e39. doi:10.1371/journal.pone.0000039, 2006.

[6] J. Lanier, You are not a gadget: A manifesto. New York: Knopf, 2010.

[7] M. M. Botvinick and J. Cohen, "Rubber hands 'feel' touch that eyes see.," Nature, vol. 391, no. 6669, p. 756, Feb. 1998.

[8] K. C. Armel and V.S. Ramachandran, "Projecting sensations to external objects: evidence from skin conductance response," Proc. R. Soc. London Ser. B-Biological Sci., vol. 270, no. 1523, pp. 1499-1506, Jul. 2003.

[9] M. Slater, D. Perez-Marcos, H. H. Ehrsson, and M. Sanchez-Vives, "Towards a digital body: The virtual arm illusion," Front. Hum. Neurosci., vol. 2, no. 6, p. doi:10.3389/neuro.09.006.2008, 2008.

[10] V. I. Petkova and H. H. Ehrsson, "If I Were You : Perceptual Illusion of Body Swapping," PLoS One, vol. 3, no. 12, p. e3832, 2008.

[11] V. I. Petkova, M. Khoshnevis, and H. H. Ehrsson, "The perspective matters! Multisensory integration in ego-centric reference frames determines full-body ownership," Front. Psychol., vol. 2, p. Article 35, 2011.

[12] B. Spanlang, X. Navarro, J.-M. Normand, S. Kishore, R. Pizarro, and M. Slater, "Real time whole body motion mapping for avatars and robots," in Proceedings of the 19th ACM Symposium on Virtual Reality Software and Technology - VRST '13, 2013, p. 175.

[13] N. Yee and J. N. Bailenson, "The Proteus effect: The effect of transformed self-representation on behavior," Hum. Commun. Res., vol. 33, no. 3, pp. 271-290, 2007.

[14] K. Kilteni, I. Bergstrom, and M. Slater, "Drumming in immersive virtual reality: the body shapes the way we play.," IEEE Trans. Vis. Comput. Graph., vol. 19, no. 4, pp. 597-605, Apr. 2013.

[15] T. Peck, S. Seinfeld, S. Aglioti, and M. Slater, "Putting yourself in the skin of a black avatar reduces implicit racial bias," Conscious. Cogn., vol. 22, no. 3, pp. 779-787, 2013.

[16] A. G. Greenwald, D. E. McGhee, and J. L. K. Schwartz, "Measuring individual differences in implicit cognition: The implicit association test," J. Pers. Soc. Psychol., vol. 74, no. 6, p. 1464, 1998.

[17] M. Martini, D. Perez-Marcos, and M. V Sanchez-Vives, "What Color is My Arm? Changes in Skin Color of an Embodied Virtual Arm Modulates Pain Threshold.," Front. Hum. Neurosci., vol. 7, p. 438, Jan. 2013.

[18] M. Martini, D. Perez-Marcos, and M. V Sanchez-Vives, "Modulation of pain threshold by virtual body ownership.," Eur.J. Pain, Jan. 2014.

[19] O. Cohen, S. Druon, S. Lengagne, A. Mendelsohn, R. Malach, A. Kheddar, and D. Friedman, "fMRI robotic embodiment: A pilot study," Fourth IEEE RAS/EMBS Int. Conf. Biometical Robot. Biomechatronics, pp. 314-219, 2012. 
Mel Slater is ICREA Research Professor at the University of Barcelona, Spain, where he coleads the Event (Experimental Virtual Environments for Neuroscience and Technology) Lab. He also holds a part-time position as Professor of Virtual Environments, University College London, UK. His research interests include virtual reality and its applications in psychology and neuroscience. He has a DSc from University of London and he can be contacted at melslater@ub.edu.

EVENT Lab

Universitat de Barcelona

Facultat de Psicologia,

Departament de Personalitat, Avaluació i Tractaments Psicològics, Campus de Mundet - Edifici Teatre, Passeig de la Vall d'Hebron 171, 08035 Barcelona

Spain.

Maria V. Sanchez-Vives is ICREA Research Professor at IDIBAPS (Institut d'Investigacions Biomèdiques August Pi i Sunyer) in Barcelona, Spain, where she is head of the Systems Neuroscience group. She is Adjunct Professor at the Faculty of Psychology at the University of Barcelona, where she is co-director of the Event (Experimental Virtual Environments in Neuroscience and Technology) Lab. She holds an MD and a PhD in neuroscience at the Institute of Neuroscience in Alicante, Spain. She can be contacted at msanche3@clinic.ub.es

Institut d'Investigacions Biomèdiques August Pi i Sunyer,

Rosselló149-153,

Barcelona 08036,

Spain. 